\title{
Diet, Exercise and Motivation in Weight Reduction: The Role of Psychological Capital and Stress
}

\author{
Mary Sophia Barnes and Tony Cassidy* \\ School of Psychology, Ulster University, Coleraine, Northern Ireland
}

Submission: October 26, 2018; Published: December 10, 2018

*Corresponding author: Tony Cassidy, School of Psychology, Ulster University, Coleraine, Northern Ireland

\begin{abstract}
This study tested the impact of diet, exercise and motivation on weight loss, stress, and health behaviour over 12 weeks, and also the predictability of the composite factor of psychological capital (self-efficacy, optimism, hope and resilience) for outcomes. 241 females between the ages of 18-35 years attending university who wanted to lose weight were recruited at week one. 141 remained at week 12. Participants were allocated to one of three groups, group 1, diet only, group 2, diet and exercise, and group 3, diet exercise and motivational interviewing (MI). Across all measures, group 3 (diet, MAA and MI) showed greatest effect, as well as for weight loss. PsyCap was not found to be a predictor of success, however, it was found to have significantly improved for group 3 at time 2. Implications for weight loss interventions for females experiencing stress at periods of low support are discussed.
\end{abstract}

Keywords: Motivational interviewing; Diet; Exercise; Psychological Capital; Stress

\section{Introduction}

Prevalence of overweight and obesity in populations, and associated comorbid diseases such as diabetes mellitus, osteoarthritis, coronary heart disease, hypertension, and stroke are now so great as to have overtaken undernutrition and infectious diseases as the leading cause of ill-health in the twentyfirst century [1,2]. In the US, two-thirds of the population are overweight and obese, and in Ireland thirty-nine percent of the adult population were found to be overweight, with eighteen percent obese [3].

The economic costs and burdens of obesity include costs to service providers, the individuals themselves, the opportunity cost to the individual, and indirect costs resulting from poor health, and premature death [4]. The social cost of obesity is found in prejudice directed at persons with obesity resulting in the unequal treatment in relation to access to healthcare, education, and employment [3]. Also, the stigmatisation of persons with obesity has been found to result in negative psychosocial behaviours such as social avoidance [3]. There is, therefore, a pressing need to treat overweight and obesity so as to prevent the many direct, and indirect long-terms costs to individuals, and their health. It is imperative that individuals at risk of overweight and obesity are encouraged to access interventions to reduce weight as early as possible in the lifespan in order to prevent detrimental consequences to health and well-being over the long-term.

A review of weight management interventions found that inclusion of routine physical activity is essential for both shortterm and long-term weight loss but only when combined with calorie restriction [4]. Behaviour change techniques in weightloss treatments aim to improve coping, and focus on changing eating behaviours, activity levels, and thinking patterns that contribute to obesity [5].

It was found that the treatments of diet restriction, exercise, and behaviour change when combined, result in a clinically significant $10 \%$ reduction in weight in the short-term, and more significantly, a reduction in binge eating and depression in women. One such technique utilised for behaviour change is that of Motivational Interviewing (MI). MI is a collaborative, supportive, client centred practice, dedicated to improving motivation and commitment to behavioural change by encouraging the setting and implementation of goals [6].

It has largely been utilised in treating substance abuse disorders but has also been integrated successfully into weight management programmes with a reduction in drop-out rates compared with standard self-help interventions [6]. A systematic review and meta-analysis of $\mathrm{MI}$ in randomised controlled trials found significant differences in weight loss for those in the MI group compared with controls [7] Booth, Prevost and found that weight management interventions in the UK were largely accessed by women, older patients, and those with comorbid conditions, and that follow up intervention was infrequent. The growing burden that overweight and obesity present requires targeted and early interventions for groups identified as being at risk for developing these conditions.

One such group are students attending university [8] first year in particular as a "critical period for weight gain". Twentyfive percent of first year students were found to have gained a clinically significant $2.3 \mathrm{~kg}$ during the first semester, with a doubling in numbers gaining weight during the second semester 
[8] Lifestyle changes identified as contributing to weight gain include, consumption of high-fat foods instead of fruit and vegetables, neglect of exercise, and increased alcohol intake [9]. Psychosocial pressures as a result of moving away from home and established social supports, pressure to develop new friendships, and academic pressures also increasing stress levels impact on weight gain most notably in female students $[9,10]$ found that social support helped males transitioning to college to moderate stress eating, but not females, as females required closer more established support compared to males.

There is a long and substantial literature on stress and coping [11] which has more recently focused on a resource approach based on the construct of psychological capital [12]. Traditionally health psychology has focused on the deficit model of stress and how health is compromised by stress [11]. However, the resource model, rooted in positive psychology, proposes that stressful events can be mediated by psychological variables such as psychological capital, social support, benefit finding, and positive health or wellbeing $[11,13]$ composite factor of psychological capital connecting hope, optimism, resilience, and self-efficacy represents what the authors describe as "an individual's appraisal of circumstances and probability for success based on motivated effort and perseverance".

The effect of stress and emotional eating has been found to be greater in women, is a contributor to the diversity in individual differences in success rates during weight loss interventions and has been found to be a predictor of weight gain amongst students, most notably females $[10,14-16]$ set out to identify the factors that separate males and females who overeat during stress from those who do not. Although no gender differences were identified with respect to intake, women compared with men were found to demonstrate greater impulsivity to eat during stressful periods as a consequence of disinhibition in disregarding self-imposed rules, as measured on the Eating Inventory scale [17].

Furthermore, women who had reported disordered eating behaviours, and more severe binging episodes were found to overeat during stressful experiences. [18] found that predictors of stress-related eating were different for men and women. Men were found to be more at risk when single, divorced and unemployed, and were more likely to consume alcohol as the level of stress eating and drinking increased. Women were found to be at greater risk due to low levels of emotional support. Furthermore, women who craved chocolate were identified as having strongly negative mood states, and as a consequence were more inclined to stress eat and develop obesity [18]

The role of stress as a significant contributor to overweight and obesity cannot be ignored as it is demonstrated to trigger the binge eating of unhealthy foods strongly related to obesity [18]. Given, therefore, that women are at greater risk of weight gain as a result of impulsive stress and emotional eating, most notably during periods lacking in emotional support, programmes designed to prevent and treat obesity should be targeted and include strategies to assist better emotional coping through development of cognitive restraint. Evidence supports the view that weight management is complex. Understanding the multiplicity of variables and individual differences in perceived stress, psychological capital, lifestyle and resilience is essential to improving outcomes for weight loss and maintenance of a healthy weight. This study, therefore, will target female students attending university, as this is a period of intense psychosocial pressure. Students will be allocated to three different weight management programmes, diet only, diet and exercise, and diet, exercise and MI, to identify which type of intervention is most likely to deliver success in weight loss through improvements in lifestyle, stress management, psychological capital and resilience. It is expected that MI will have the most significant effect on the measured outcomes.

This study seeks to test the impact of three different interventions (diet information only, diet information and moderate aerobic activity, and diet information, moderate aerobic activity and motivational interviewing) on weight loss, stress, and health behaviour over 12 weeks, and to test if psychological capital (self-efficacy, optimism, hope and resilience) would be predictive of outcome.

\section{Methods}

\section{Design}

This is a short-term longitudinal randomised control intervention using 3 (conditions) x 2 (time-points) design. The first outcome variable will be weight lost, and the predictor variable is Psychological Capital (PsyCap).

\section{Participants and Procedure}

A total of 241 female students between the ages of 18-25 years responded to an e-mail circulated to 2,300 students via the Ulster email system. The e-mail sought to recruit females between the ages of 18-25 years who wanted to lost weight. Exclusion criteria were those aged under 18 or over 25 , those already engaged in a weight loss intervention, males, and those with no desire to lose weight. A link to an online questionnaire was emailed to all 241 responders. Using height and the weight screening tool of Body Mass Index (BMI) scores, participants were randomly allocated to one of 3 conditions: A) diet information only, B) diet information and MAA, and C) diet information, MAA and a motivational interviewing session.

A PDF attachment providing dietary information and guidance was sent to all participants (Appendix 2.1). This was repeated at 3-week intervals for a total of 12 weeks. Additionally, participants in conditions $\mathrm{B}$ and $\mathrm{C}$ were supplied with information on the benefits of moderate aerobic activity (MAA), and how to achieve it, and were requested to record a minimum of 150 minutes of MAA per week. Over each of the individual 12 weeks, participants were contacted via email which asked for details of minutes recorded for MAA that week. Participants in condition C were invited to attend a 3-hour group based Motivational Interviewing session and were provided with a questionnaire facilitating selfreflection based on motivational principles every third week. All 
participants were assessed before the intervention and also at the end of the 12 weeks on the measures below. By the end of the 12 weeks, only 141 participants remained, 42 in group 1, 32 in group 2 , and 67 in group 3.

\section{Measures}

A survey questionnaire was constructed including a section on demographics requesting information regarding, age, height, and weight, and the following measures:

\section{Perceived stress}

The Perceived Stress Scale - 10 item [19], developed from the 14 item Perceived Stress Scale (PSS) [19] is a measure of how unpredictable, uncontrollable, and overloaded an individual perceives their life to be (Appendix 5). Based on the theory of stress appraisal [20] the PSS is a measure of the "degree to which situations in one's life are appraised as stressful [19]. Internal consistency for the PSS was strong $(\alpha=.86)$.

\section{Psychological capital (PsyCap)}

This composite construct consisting of the four factors of hope, resilience, optimism, and self-efficacy has been utilised as a psychometric tool to predict work performance and job satisfaction [13]. PsyCap is described as a "positive state-like capacity" that can be positively enhanced through support [20] The study used the PCQ-24 measure $(\alpha=.89)$ [20].

All four component factors were measured using 6 items. Participants were asked to rate how they feel about themselves in order to establish their state-like capacity at that moment. For example, self-efficacy ( $\alpha=.85$ ): 'I feel confident helping to set targets/goals in my work area'; hope $(\alpha=.80)$ :'I can think of many ways to reach my current work goals'; resilience $(\alpha=.72)$ : 'I can usually take stressful things at work in stride'; optimism $(\alpha=.79)$ : 'When things are uncertain for me at work, I usually expect the best'. The Self-Rater Version was used with agreement rated on a 6 -point Likert-type scale $(1=$ strongly disagree to $6=$ strongly agree).

\section{Health Action}

This was measured using the 15 items on the Reported Health Behaviour Checklist which assesses lifestyle behaviours that impact on health, e.g. sleep, diet, and exercise.

\section{Ethical Considerations}

Permission to conduct the study was granted by the Ulster University Filter Committee. The study was designed in accordance with the British Psychological Society's [21] and the ethical standards of Ulster University for research with human participants.

\section{Data Analysis}

Following completion of data collection, the four scales for each participant were scored and entered onto SPSS for analysis. Descriptive statistics were examined to identify the mean and standard deviation for each group and each of the four measures at both time 1 and time 2. A mixed model Anova with repeated measures across time and between participants for the three conditions was conducted to test for main effects and interactions. A one-way Anova was used to test for baseline differences between the groups and the four outcome variables.

\section{Results}

This study tested the effect of three different weight loss interventions on weight loss, stress and health behaviour over 12 weeks, and tested whether psychological capital is predictive of outcome. The first stage of analysis resulted in the calculation of descriptive statistics for stress, health action, weight and psychological capital by group and time, and are outlined in Table 1.

Table 1: Descriptive statistics for stress, health action, weight, and psychological capital by group and time.

\begin{tabular}{|c|c|c|c|c|c|c|c|c|c|}
\hline Group & & & & Health & Health & Weight & Weight & & \\
\hline & & Stress & Stress & Action & Action & in lbs. & in lbs. & PsyCap & PsyCap \\
\hline & & 1 & 2 & 1 & 2 & 1 & 2 & 1 & 2 \\
\hline \multirow[t]{2}{*}{ Diet only } & Mean & 3.16 & 3.11 & 3.05 & 3.16 & 176.74 & 173.87 & 12.03 & 12.18 \\
\hline & SD & 1.13 & 1.09 & 1.31 & 1.286 & 27.7 & 29.94 & 3.48 & 3.39 \\
\hline Diet \& & Mean & 2.76 & 2.05 & 3.51 & 4.49 & 176.34 & 156.12 & 11.63 & 13.02 \\
\hline Exercise & SD & 1.09 & 0.92 & 1.12 & 0.78 & 37.85 & 22.85 & 2.36 & 1.88 \\
\hline Diet, & Mean & 2.97 & 1.94 & 3.23 & 4.23 & 175.15 & 149.53 & 11.69 & 13.31 \\
\hline $\begin{array}{l}\text { Exercise \& } \\
\text { Motivational } \\
\text { Interview }\end{array}$ & SD & 1.06 & 0.85 & 1.35 & 0.93 & 26.67 & 23.45 & 2.54 & 2.29 \\
\hline
\end{tabular}

The second stage of analysis was to use a mixed model Anova to test for main effects and interactions with repeated measures across time, and between participants on the three conditions. For stress at times one and two there was a significant main effect $\left(\mathrm{F}(1,138)=14.688, \mathrm{P}<.001, n p^{2}=.096\right)$. The partial eta squared $\left(n p^{2}\right)$ of .096 is between a medium and large effect size. Cohen's
(1988) rule of thumb suggests that $0.01=$ small effect, 0.06 $=$ medium effect, and $0.14=$ large effect. A significant stress by group interaction was also found $\left(\mathrm{F}(2,138)=4.158, \mathrm{p}<.05, n p^{2}\right.$ $=.057)$, and the partial eta squared $\left(n p^{2}\right)$ of .057 falls between medium and large effect. Mixed Anova was again used to test weight at times one and two. 
A significant main resulted $\left(\mathrm{F}(1,138)=77.812, \mathrm{p}<.001, n p^{2}\right.$ $=.36)$. The partial eta squared $\left(n p^{2}\right)$ of .36 shows a large effect size. A significant weight by group interaction also resulted (F (2, 138) $=21.837, \mathrm{p}<.001, n p^{2}=.24$ ). Mixed Anova was also used to test health action at times one and two. There was a significant main effect $\left(\mathrm{F}(1,138)=35.97, \mathrm{p}<.01, n p^{2}=.21\right)$. The partial eta squared $\left(n p^{2}\right)$ of .21 demonstrates a large effect size. Health action by group interaction was also significant $(\mathrm{F}(2,138)=5.439, \mathrm{p}<.01$, $\left.n p^{2}=.08\right)$.

Mixed Anova was also used to test psychological capital at times one and two. There was a significant main effect $(F(1,138)$ $\left.=21.05, \mathrm{p}<.001, n p^{2}=.13\right)$. The partial eta squared $\left(n p^{2}\right)$ of .13 demonstrates a medium effect size. Psychological capital by group interaction was not significant. As 241 participants had started, and only 141 completed the programme, a Chi-square test was conducted to test any relationship between completing and the experimental condition. A significant relationship resulted (chisquare $(2)=19.718, p<.001$ ) demonstrating that participants in the group of combined diet, exercise, and motivational interviewing were more likely to complete the programme.

In order to check for baseline, bias a one-way Anova was used to test for differences between the groups on stress, health action, psychological capital and weight at time one. There were no main effects. one-way Anova between the groups for stress, health action, psychological capital and weight at time two show main effects for stress $(\mathrm{F}(2,138)=17.21, \mathrm{p}<.001)$, health action $(\mathrm{F}(2,138)=21.99, \mathrm{p}<.001)$, psychological capital $(\mathrm{F}(2,138)=6.29$, $\mathrm{p}<.01)$, and weight $(\mathrm{F}(2,138)=7.43, \mathrm{p}<.001)$. In addition weight loss was calculated by subtracting weight at time 2 from weight at time 1 and one-way Anova across groups showed a main effect for group $(\mathrm{F}(2,138)=29.13, \mathrm{p}<.001)$.

\section{Discussion}

This study tested the impact of three different interventions for three groups, [14] diet information only, [8] diet information and moderate aerobic activity, and [3] diet information, moderate aerobic activity, and motivational interviewing, on weight loss, stress, and health behaviour over twelve weeks. Also tested was whether the composite factor of psychological capital would be predictive of outcome. No differences were found between the groups on weight, stress, health action and psychological capital at the commencement of the study.

In line with expectations, a main effect for each of the three groups was found between times one and two for weight, stress, and health action, with the motivational interviewing condition showing the greatest effect for these measures. Furthermore, it was found that those allocated to this condition were more likely to complete the programme. In particular, results show a significant effect for weight loss in each of the three groups. Weight loss differences by group were also observed, with the most significant weight loss occurring in the MI condition, followed by group two, diet information and MAA. The least amount of weight lost was observed in group one, diet only. The finding of greater weight loss for the MI condition is consistent with a number of reviews which have found that behavioural approaches to weight control result in more successful and sustained weight loss [7,20-25].

Behaviour programmes restricting dietary intake, undertaking regular exercise of between 150-200 minutes minimum per week, and self-monitoring as conducted in this study for groups two and three, have been identified as being associated with improved weight loss [4]. Furthermore, the provision of dietary information and guidance at three weekly intervals over the twelve weeks to each of the three groups in this study likely encouraged positive cues for eating healthy food for all participants. Such cues are found to encourage the removal of high fat/high calorie foods from places of residence and increase positive behaviours around the provision of low-calorie alternatives [25-32]. This could explain why weight loss was significant for all three groups.

Equally, the provision of information on the benefits of MAA in its different forms, the recording of MAA by groups two and three, and the weekly recording of MAA by email is supported by evidence for the combination of diet and exercise for successful weight loss, over exercise alone, [4]. The inclusion of selfmonitoring further promotes more successful weight loss [4]. Groups two and three undertook and recorded a minimum of 150 minutes of exercise per week. However, participants were not directed as to how often to exercise. [4] review examined the question as to whether several short bursts of exercise were more efficient for weight loss compared with one long bout of exercise, finding that short bursts were as beneficial for weight loss as one long bout of exercise. Thus, it is likely that any differences in the scheduling of exercise were unlikely to have interfered with results. Rather, it is the inclusion of exercise as a lifestyle change that is beneficial for weight loss. The inclusion of motivational interviewing (MI) resulted in a significant difference in weight loss for this group. Weight loss was twice that achieved in group two, and eight times more than that in group one (Table 1). This finding is consistent with [7] review which identified MI as having a medium effect on weight loss. [7] assert that the directive, patient-centred counselling approach of MI is appropriate for weight loss programmes as it promotes greater focus on the goal of weight loss. Although MI has most commonly been used in the field of addictions to motivate for change, and to promote adherence, its usefulness in weight loss is focused on behaviour modification rather than on the cessation or total abstinence from a substance [7]. There is, therefore, a difference in how it is applied for weight loss. Furthermore, [7] found that MI and behavioural interventions combined tended to result in greater weight loss than for minimal intervention control groups. Again, this finding is consistent with the present study. The centrality of weight loss as the principal goal as opposed to a fitness goal, a dietary goal, or a health status change goal such as lowered cholesterol is key to greater weight loss [7].

MI has also been found to promote adherence, a strong predictor of weight loss [7]. In this study, there was a significant finding for participants in the experimental group completing the programme and achieving greater weight loss. It could, therefore, be concluded that adherence in terms of diet, exercise, and the 
setting of goals all improved due to the MI. The inclusion of MI in the experimental condition certainly resulted in greater weight loss, and significant effects across all measures.

A significant effect for both stress and health action was found at times one and two over the twelve weeks, and a significant effect for stress and health action by group with the greatest reduction in stress observed in the experimental condition. The next greatest reduction in stress occurred in group two, followed by group one. The bi-directional relationship between stress and the consumption of high calorie foods in females during periods of high stress is well documented [16]. Binge eating can be a serious confounder for weight loss. As participants in this study were all females attending university, a period of time associated with low emotional support, it is noteworthy that participants in group 3 (MI) experienced the greatest reduction in stress, and highest scores for positive health behaviours. This indicates that both health behaviour and adherence were improved by MI.

The second question asked pertained to the predictability of the composite factor of psychological capital (PsyCap), made up of hope, resilience, optimism, and efficacy. As there was no significant difference between the groups for psychological capital, it was found not to be a predictor of success. There was, however, a significant main effect for improvement in this statelike construct between times one and two for the experimental condition, with a medium effect for group two. The rationale for using PsyCap as a predictor of success was due to this construct's usefulness in identifying resources within individuals which would improve that individual's positive appraisal of circumstances, thereby improving the probability of success [13]. The state-like element demonstrated effect. The predictability of the measure did not. Furthermore, two measures were used to test for psychological capital, the PCQ-24 and the Brief Resilience Scale (BRS). Resilience was identified as an important component of PsyCap as it measures just how strongly participants were able to recover from stressful events, adapt, and continue to adhere to the weight loss programme. Consequently, this study's design included a second measure to compliment PsyCap. The fact that greater numbers completed the study from the experimental group than from the two other groups indicates that resilience developed between times one and two However, as resilience is likely to have developed considerably as a consequence of the intervention, a question remains as to whether the inclusion of the second measure of the BRS favoured the state-like element over the predictive.

\section{Conclusion}

This study's findings could be useful for the design of targeted weight loss interventions as part of a stress management programme for females through university health centres or indeed in an occupational health setting. Supporting female university students through the adjustment of moving away from home, and social supports during the first year of university is important, as this is a period when students are vulnerable to weight gain [8] Nonetheless, longitudinal research using a more diverse sample, and similar design and measures, including one for binge eating, is needed. Such a study would test firstly, the effect of disinhibition on binge eating on adherence and weight, and secondly, test for long-term weight maintenance, and finally, test for the predictability of psychological capital.

\section{References}

1. The British Psychological Society (2010) Code of Human Research Ethics. Leicester: The British Psychological Society.

2. World Health Organisation (2000) WHO Technical Report Series 894: Obesity: Preventing and Managing the Global Epidemic. Geneva: World Health Organisation.

3. The National Taskforce on Obesity (2005) Obesity The Policy Challenges: The Report of The National Taskforce on Obesity. HSE.

4. Pinto AM, Gokee-LaRose J, Wing R (2007) Behavioral Approaches to Weight Control: A Review of Current Research. Women's Health 3(3): 341-53.

5. Krejci J, Neugebauer Q (2015) Motivational Interviewing in Groups: Group Process Considerations. Journal of Groups in Addiction and Recovery 23-40.

6. Armstrong M, Mottershead T, Ronksley P, Sigal R, Campbell T, et al. (2011) Obesity Reviews. 709-723.

7. Anderson DA, ShapiroJR, Lundgren JD (2003) The Freshman Year of College as a Critical Period for Weight Gain: An Initial Evaluation. Eat Behav 4(4): 363-367.

8. Serlachius A, Hamer M, Wardle J (2007) Stress and Weight Change in University Students in the United Kingdom. Physiol Behav 92(4): 54853.

9. Darling KE, Fahrenkamp AJ, Wilson SM, Karazasia BT, Sato AF (2017) Does Social Support Buffer the Association Between Stress Eating and Weight Gain During the Transition to College? Differences by Gender. Behav Modif 41(3): 368-381.

10. Cassidy T (2011) Stress, Coping, Resilience, and Health. In Managing Stress: From Theory to Application NY: Nova Science, USA pp. 1-40.

11. Cassidy T, Giles M, McLaughlin M (2014) Psychosocial Capital and Health: A Resource Model of Stress Using Structural Equation Modelling. Progress in Psychology 11-17.

12. Luthans F, Avolio b, Avey J, Norman S (2007) Positive Psychological Capital: Measurement and Relationship with Performance and Satisfaction. Personnel Psychology 541-572.

13. Adam TC, Epel ES (2007) Stress, Eating and the Reward System. Physiol Behav 91(4): 449-58.

14. Goldbacher E, La Grotte C, Komaroff E, Vander Veur S, Foster GD (2016) An Initial Evaluation of a Weight Loss Intervention for Individuals who Engage in Emotional Eating. J Behav Med 39(1): 139-150.

15. Weinstein SE, Shide DJ, Rolls BJ (1997) Changes in Food Intake in Response to Stress in Men and Women: Psychological Factors. Appetite 28(1): 7-18.

16. Stunkard A, Messick S (1985) The Three-Factor Eating Questionnaire to Measure Dietary Restraint, Disinhibition and Hunger. Journal of Psychosomatic Research 29(1): 71-83.

17. Laitinen J, Ek E, Sovio U (2002) Stress-Related Eating and Drinking Behavior and Body Mass Index and Predictors of this Behavior. Preventive Medicine 34(1): 29-39.

18. Cohen J (1988) Statistical Power Analysis for the Behavioral Sciences (2nd ed.) Hillsdale. New York L Erlbaum Associates USA.

19. Lazarus RS, Folkman S (1984) Coping and Adaption. In The Handbook of Behavioral Medicine pp. 282-325. 
20. Booth H, A Prevost T, Gulliford CM (2015) Access to Weight Reduction Interventions for Overweight and Obese Patients in UK Primary Care: Population-Based Cohort Study pp. 1-6.

21. Cohen S, Williamson G, S Spacapam, S. oskamp, GM Williamson et al. (1988) Perceived Stress in a Probability Sample of the United States. Soc Psychol Heal Claremont Symp Appl Soc Psychol Newbury Park CA Sage.

22. Di Marco I, Klein D, Clark V, Wilson T (2009) The Use of Motivational Interviewing Techniques to Enhance the Efficacy of Guided Self-Help Behavioral Weight Loss Treatment. Eating Behaviors 10(2): 134-136.

23. Herman C, Polivy J (1980) Restrained Eating. In: Stunkard A (Ed.) Obesity, pp. 208-225.

24. Herman C, Polivy J (1980) Restrained Eating. In Stunkard A (Ed.), Obesity (pp. 208-225). Philadelphia: Saunders.

25. Luthans F, Youssef C, Avolio B (2007) Psychological Capital. New York: Oxford University Press, USA.

26. Morrison V, Bennett P (2012). An introduction to Health Psychology (2nd edn.). Essex UK: Pearson Prentice Hall.
27. Smith B, Dalen J, Wiggins K, Tooley E, Christopher P et al. (2008) The brief resilience scale: assessing the ability to bounce back. Int J Behav Med 15(3): 194-200.

28. Spielberger C, Gorsuch R, Lushene R, Vagg P, Jacobs G (1983) Manual for the State-Trait Anxiety Inventory (Form Y) (Self-Evaluation Questionnaire). Palo Alto, CA: Consulting Psychologists Press, Inc.

29. University of Ulster (2nd.) Data Handling Procedures/Guidance.

30. Weinstein SE, Shide DJ, Rolls BJ (1997) Changes in Food Intake in Response to Stress in Men and Women: Psychological Factors. Appetite 28(1): 7-18

31. World Health Organisation (2015) Obesity and overweight. World Health Organisation.

32. Yates EA, MacPherson AK, Kuk JL (2012) Secular trends in the diagnosis and treatment of ovesity among US adults in the primary care setting. Obesity 20(9): 1909-1914.

\section{Your next submission with Juniper Publishers} will reach you the below assets

- Quality Editorial service

- Swift Peer Review

- Reprints availability

- E-prints Service

- Manuscript Podcast for convenient understanding

- Global attainment for your research

- Manuscript accessibility in different formats

(Pdf, E-pub, Full Text, Audio)

- Unceasing customer service

\section{Track the below URL for one-step submission}

https://juniperpublishers.com/online-submission.php 